\title{
La collection de vases grecs du marquis de Northampton (1790-1851). Entre archéologie et sciences de la nature
}

The collection of Greek vases belonging to the Marquess of Northampton (17901851). Between archaeology and the natural sciences

\section{Marie-Amélie Bernard}

\section{OpenEdition}

Journals

Édition électronique

URL : http://journals.openedition.org/cel/396

DOI : 10.4000/cel.396

ISSN : 2262-208X

Éditeur

École du Louvre

Édition imprimée

Date de publication : 1 octobre 2014

Référence électronique

Marie-Amélie Bernard, «La collection de vases grecs du marquis de Northampton (1790-1851). Entre archéologie et sciences de la nature », Les Cahiers de l'École du Louvre [En ligne], 5 | 2014, mis en ligne le 01 octobre 2014, consulté le 15 novembre 2019. URL : http://journals.openedition.org/cel/396 ; DOI : $10.4000 /$ cel.396

\section{(c) (†) $\odot$}

Les Cahiers de l'École du Louvre sont mis à disposition selon les termes de la licence Creative Commons Attribution - Pas d'Utilisation Commerciale - Pas de Modification 4.0 International. 


\title{
Cahiers de l'École du Louvre
} recherches en histoire de l'art, histoire des civilisations archéologie, anthropologie et muséologie

\section{Numéro 5. Octobre 2014}

\author{
La collection de vases grecs \\ du marquis de Northampton (1790-1851). \\ Entre archéologie et sciences de la nature \\ Marie-Amélie Bernard
}

Article disponible en ligne à l'adresse :

http://www.ecoledulouvre.fr/cahiers-de-I-ecole-du-louvre/numero5octobre2014/Bernard.pdf

Pour citer cet article :

Marie-Amélie Bernard, « La collection de vases grecs du marquis de Northampton (1790-1851). Entre archéologie et sciences de la nature », Cahiers de l'École du Louvre. Recherches en histoire de l'art, histoire des civilisations, archéologie, anthropologie et muséologie [en ligne] no 5, octobre 2014, p. 4 à 14.

\section{@creative \\ (요 $\odot$}

(C) École du Louvre

Cet article est mis à disposition selon les termes de la Licence Creative Commons Attribution - Pas d'utilisation commerciale - Pas de modification 3.0 non transposé. 


\section{Cahiers de l'École du Louvre recherches en histoire de l'art, histoire des civilisations archéologie, anthropologie et muséologie \\ Numéro 5. octobre 2014}

\section{Sommaire}

\section{Introduction}

L'archéologie en construction : objets, images, dispositions

Nathan Schlanger

La collection de vases grecs du marquis de Northampton (1790-1851).

Entre archéologie et sciences de la nature

Marie-Amélie Bernard

L'artisanat touristique du Sud-Ouest des États-Unis. L'exemple des objets collectés par Alphonse Pinart à Santa Fe, à la fin du XIX siècle Éloïse Galliard

Regard sur les statuettes hindoues et bouddhiques en bronze d'Indonésie. Leur rôle pour la connaissance de la civilisation javanaise ancienne et ses liens avec l'Asie du Sud et du Sud-Est

Mathilde Mechling p. $25-33$

Le Tigre, le Louvre et l'échange de connaissances archéologiques visuelles entre la France et la Grande-Bretagne aux alentours de 1850

Mirjam Brusius p. $34-46$

Le regard des photographes commerciaux. Quelques clichés du fonds égyptien de la Collection Fouad Debbas à l'étude.

Yasmine Chemali - Anne-Hélène Perrot

Émile Guimet et la morsure du canard égyptien.

Un curieux au musée de Boulaq

Thomas Lebée

L'exposition préhistorique de la Galerie de l'Histoire du travail en 1867.

Organisation, réception et impacts

Charlotte Quiblier. 


\section{La collection de vases grecs \\ du marquis de Northampton (1790-1851). Entre archéologie et sciences de la nature}

\section{Marie-Amélie Bernard}

Amateur averti et fortuné, Spencer Joshua Alwyne Compton, deuxième marquis de Northampton, forma entre les années 1820 et 1850 une collection de vases grecs restreinte mais attentivement choisie dont la constitution permet de problématiser le passage de l'antiquarisme à l'archéologie. Vers 1820, s'installa à Rome un cercle d'historiens, d'archéologues, d'érudits et d'artistes qui se passionnaient pour l'étude de l'Antiquité. Ce premier cénacle, le Cercle des Hyberboréens, fut remplacé en 1828 par l'Instituto di Corrispondenza Archeologica : animé en premier lieu par Eduard Gerhard (1795-1868), l'Instituto était un organisme européen de diffusion et d'étude des découvertes archéologiques, où collaboraient savants, érudits et collectionneurs. Il joua un rôle essentiel dans l'activité archéologique de la première moitié du XIX ${ }^{e}$ siècle notamment par ses publications, le Bulletino, les Annali et les Monumenti Inediti ${ }^{2}$. Cette période fut aussi celle de vifs questionnements sur les rapports entre les sciences de l'homme et les sciences de la nature ${ }^{3}$. Alors qu'au début du siècle, Georges Cuvier (1769-1832) en France ou William Buckland (17481856) en Angleterre s'étaient inspirés des méthodes des antiquaires pour faire progresser de manière considérable la géologie et la paléontologie ${ }^{4}$, la jeune archéologie regardait à son tour vers les sciences de la nature. Cette recherche de convergence des champs disciplinaires se trouva des perspectives avec la parution, en 1847, du premier volume des Antiquités celtiques et antédiluviennes dans lequel Jacques Boucher de Perthes (1788-1868) proposa une synthèse des sciences de la nature et des sciences de l'homme'.

Dans les années 1820, Spencer Joshua Alwyne Compton s'installa à Rome où le climat de ferveur archéologique qui régnait alors fut propre à susciter chez lui le désir de collectionner. Trois décennies plus tard, les dernières années du marquis se déroulèrent dans une ambiance totalement différente : la fin des années 1840 fut marquée par une réforme de la Royal Society, qui, auparavant composée aussi bien de savants que de riches amateurs, se dota de règles visant à un recrutement plus scientifique et plus professionnel. Northampton en était alors le président et, opposé à la réforme, il démissionna en 1848 , concrétisant par sa décision l'entrée dans un nouveau moment de l'histoire des sciences ${ }^{6}$. Cette moitié du siècle vit aussi la publication, en 1850, des Archäologische Thesen d'Eduard Gerhard ${ }^{7}$, autre tournant scientifique qui entérina la distance prise par l'archéologie avec les recherches des antiquaires et les plaisirs de l'esthète.

Ce fut donc pendant une période fondamentale pour l'histoire des idées que Lord Northampton bâtit sa collection. Son rang, tout comme la qualité des vases qu'il a su réunir pourraient laisser penser, à première vue, qu'il ne s'agit que de la très belle collection d'un riche aristocrate. Mais un examen attentif soulève des interrogations plus larges sur le choix des pièces : les formes sont variées à

1. L'auteur remercie Nathan Schlanger et Anne Ritz-Guilbert qui lui ont suggéré cet article à la suite d'une présentation de sa thèse en cours « Francesco Depoletti, marchand et restaurateur de vases grecs (1779-1854) », dirigée par Brigitte Bourgeois (C2RMF - École du Louvre) et Alain Schnapp (Université Paris 1 - Panthéon Sorbonne). Elle remercie également Martine Denoyelle et François Lissarrague pour leur aide.

2. Alain Schnapp, La Conquête du passé, Paris, Carré, 1993, p. 376.

3. Sur ce sujet voir A. Schnapp, «L'invention de I'archéologie », op. cit. note 2, pp. 333-387, et en particulier la section intitulée «Penser l'archéologie comme une science de la nature », p. 371 sq.

4. A. Schnapp, op. cit. note 2, pp. 335-352.

5. A. Schnapp, op. cit. note 2, p. 384.

6. Marie Boas Hall, All scientists now. The Royal Society in the Nineteenth Century, Cambridge, Cambridge university Press, 1984.

7. Eduard Gerhard, «Archäologische Thesen », dans Archäologischer Anzeiger zur Archäologischen Zeitung, VIII, 1850, pp. 203-206. Sur l'importance de ce texte, voir A. Schnapp, op. cit. note 2, p. 372. 
dessein, les choix iconographiques sont récurrents, et les fragments nombreux ; cette collection d'exception n'est-elle pas aussi le reflet et le support des réflexions d'un homme passionné par les disciplines à la naissance desquelles il assista?

\section{Spencer Joshua Alwyne Compton, marquis de Northampton (1790-1851)}

Né en 1790 Spencer Joshua Alwyne Compton (fig. 1) fit ses études au Trinity College de Cambridge. Membre du Parlement de 1812 à 1820, il épousa en 1815 Margaret Macleane Clephane (1794 ?-1830), pupille de Walter Scott qui appréciait ses talents musicaux et poétiques ${ }^{8}$. Le couple $s^{\prime}$ installa en Italie dans les années 1820 et en 1828 , à la mort de son père, celui qui était connu jusqu'alors comme le comte Compton prit le titre de marquis de Northampton. En 1830, à la suite du décès soudain de sa femme, il rentra en Angleterre avec ses enfants. En 1840, il entreprit un voyage en France, suivi par d'autres, en Italie et en Allemagne, puis en Grèce et en Égypte d'où il rapporta des antiquités qu'il offrit au British Museum?.

David Octavius Hill et Robert Adamson Spencer Joshua Alwyne Compton $2^{\text {nd }}$ Marquess of Northampton
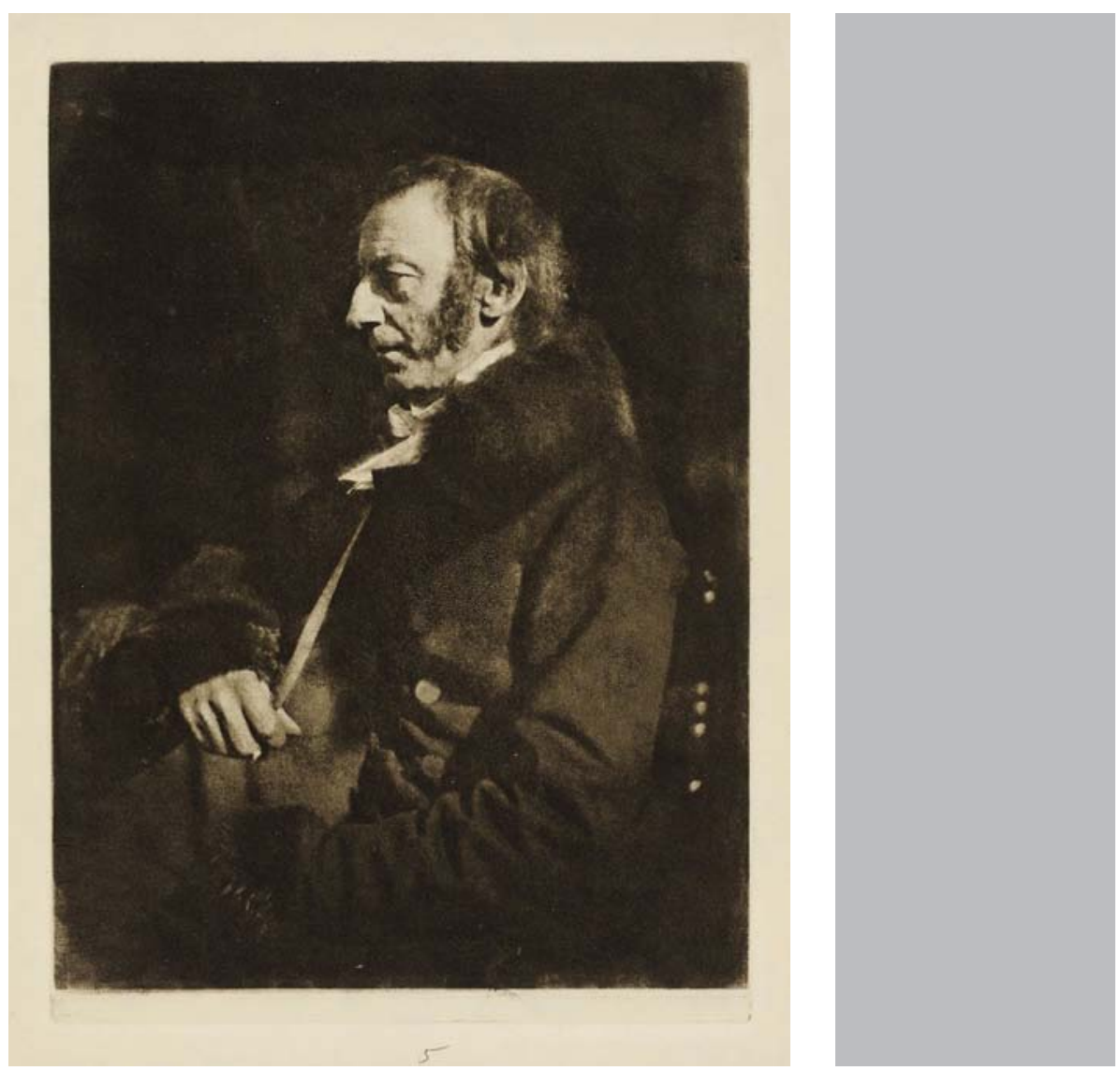

Membre de l'Instituto di Corrispondenza Archeologica dès sa fondation, Northampton joua un rôle de premier plan dans plusieurs sociétés savantes : il fut président de la Geological Society (1820-1822), de la Royal Society (18381848), de l'Archeological Institute of Great Britain and Ireland (1845-1846 puis 1850-1851), membre de la British Association for the Advancement of Science, trustee de la National Gallery et du British Museum. Son rôle dans ces institutions fut capital, moins du point de vue de ses contributions scientifiques que de ses talents d'organisateur : Lord Northampton savait susciter l'adhésion à une idée ou un projet ${ }^{10}$, créer une atmosphère propice à la collaboration intellectuelle et

8. « Obituary », The Gentleman's Magazine, 1851, p. 425.

9. Revue Archéologique 1851, p. 764. Bulletin monumental, 2e série, t. 7, vol. 17, 1851, p. 239. 10. Jack Morrell et Arnold Thackray, (ed.), Gentlement of Science. Early Correspondence of the British Association for the Advancement of Science, Londres, 1984. 
apaiser les conflits ${ }^{11}$. De plus, il recevait volontiers des savants britanniques ou étrangers dans sa demeure de Castle Ashby.

L'intérêt de Lord Northampton pour l'archéologie était bien marqué. Il visita de nombreux sites, qu'il dessina parfois. En 1823, lors d'un voyage de quatre mois en Sicile, il constitua un carnet de soixante-dix-neuf paysages monochromes, récemment exposés à Rome ${ }^{12}$. Dans les temples de Ségeste et d'Agrigente, dans les promontoires basaltiques des îles Cyclopes, nous pouvons déceler son intérêt pour le passé de l'homme et pour celui de la nature, pour l'archéologie et pour la géologie. Il voulut voir des fouilles, dont il emportait souvent un vase en souvenir, comme c'était l'usage quand une personne de qualité se rendait sur un chantier : de Nola, il rapporta une amphore à col sous laquelle il inscrivit « Found by myself at an excavation at Nola ${ }^{13}$. À Rome, il finança lui-même une fouille : en 1830 il s'associa au comte de Shrewsbury ${ }^{14}$, à Lord Lovaine ${ }^{15}$, au colonel FitzClarence ${ }^{16}$, et au chargé d'affaires de Hanovre, August Kestner ${ }^{17}$ pour demander des permis : à proximité de la Via Nomentana ${ }^{18}$, ils découvrirent plusieurs inscriptions, des sculptures de marbre et de terre cuite, des fragments d'enduit peint, etc. La même année, les 6 et 7 mars, il était à Vulci quand, dans une tombe à trois chambres, on découvrit des armes, un trépied, des vases de bronzes, et des céramiques dont une amphore attique à figures noires qui entra directement dans sa collection ${ }^{19}$. Sa place dans le milieu archéologique romain était suffisamment importante pour que Pietro Manzi (1785-1839), un personnage ambivalent qui eut une activité de fouilles extrêmement importante sur la majorité des grands sites d'Étrurie méridionale, lui dédiât une lettre archéologique consacrée à la découverte d'une tombe de Tarquinia ${ }^{20}$.

Cet intérêt pour l'archéologie n'était pas exclusif: comme Sir William Hamilton avant lui ${ }^{21}$, Lord Northampton était très versé dans les sciences de la nature, en particulier la géologie. Du fait de ses études à Cambridge et de son rôle dans les sociétés savantes, il avait dans ses relations l'élite intellectuelle britannique : des membres de l'aristocratie, engagés dans une carrière politique ou militaire, des antiquaires, des archéologues, mais aussi des spécialistes des sciences de la nature dont le botaniste et collectionneur d'antiquités Dawson Turner (17751858), le mathématicien George Peacock (1791-1858), les astronomes John William Lubbock (1803-1865) ${ }^{22}$, et John Frederick William Herschel (17921871), les géologues Roderick Murchisson (1792-1871), Adam Sedgwick (17851873) et Charles Lyell (1767-1849), les géologues et paléontologues William Buckland (1784-1856) et Gideon Mantell (1790-1852), etc.

Membre de la Geological Society, il constitua un cabinet de minéraux et de fossiles enrichi de spécimens récoltés lors de ses voyages en Italie ${ }^{23}$, ou de ses prospections en Angleterre ${ }^{24}$, et il publia trois articles ayant trait à la géologie ${ }^{25}$.

11. M. B. Hall, op. cit. note 6.

12. Viaggio in Sicilia : il taccuino di Spencer Joshua Alwyne Compton, Milan, Silvana Editoriale, 2013.

13. John Boardman, Martin Robertson, Corpus Vasorum Antiquorum Great Britain 15 Castle Asbhy, Oxford, Oxford university press, 1979 (ensuite abrégé en CVA Castle Ashby), n 92, p. 35, pl. 55.4-5.

14. John Talbot, comte de Shrewsbury.

15. Algernon George Percy, duc de Northumberland (1810-1899), appelé Lord Lovaine entre 1830 et 1865 .

16. Lord Frederick FitzClarence (1799-1854).

17. August Kestner (1777-1853)

18. Archivio di Stato di Roma, Camerlengato II, tit.IV, b. 204, f.1220 et b.205, f. 1246

19. The Literary gazette and journal of belles-lettres, arts, sciences \& $c, v$ v. 14, 1830. Sur I'amphore : CVA Castle Ashby, n 6, pl. 11.1-2 et pl. 12. John Davidson Beazley, Attic Black-figure Vase-Painters (ensuite abrégé en ABV) 329.2. Beazley Archive 301773 Page d'accueil : http://www.beazley.ox.ac.uk/index.htm

20. Pietro Manzi, Lettera a lord Northampton sopra una tomba etrusca scoperta in Corneto I'anno 1831, Rome, 1831, publiée sous forme résumée dans les Annali dell'instituto di Corrispondenza Archeologica, 1831, v. III, pp. 327-337

21. John Thackray, " "The Modern Pliny". Hamilton and Vesuvius. », Ian Jenkins, Kim Sloan et Carlo Knight, Vases and volcanoes: Sir William Hamilton and his collection, Londres, British Museum Press, 1996, p. 65-74. L'auteur souligne que, tout passionné qu'il fût par I'observation du Vésuve, Hamilton ne collectionna jamais les roches et minéraux.

22. Père du préhistorien John Lubbock (1834-1913).

23. Op. cit. note 12, p. 20 . Une partie de ce cabinet est conservé au musée de Northampton.

24. Lord Northampton fut décrit par Richard Owen comme un inlassable chercheur de fossiles.

Voir J. Morrell, « Compton, Spencer Joshua Alwyne », Oxford Dictionnary of National Biography, v. 12 , pp. $895-897$

25. Morrell, art. cit. note 24. 
Il fit une partie de son voyage en Sicile avec Charles Lyell, géologue éminent dont l'ouvrage Principles of Geology, paru entre 1830 et 1833, fit date. En 1829, Lyell lui donna des conseils pour commencer sa collection de fossiles ${ }^{26}$. En 1840, lors de son voyage dans l'ouest de la France qu'il parcourut de Rouen à Saumur, en passant par Rennes ${ }^{27}$, Lord Northampton fit une étape à Caen et y rencontra Arcisse de Caumont (1801-1873). Ce dernier, antiquaire formé aux sciences de la nature, et en particulier à la botanique et à la géologie ${ }^{28}$, témoigna de cet intérêt pour une enquête du sol :

"Nous avons vu M. le marquis de Northampton à Caen il y a une dizaine d'années ; l'archéologie et la géologie l'occupaient particulièrement dans ce voyage. Il était accompagné de plusieurs secrétaires, et fit diverses courses dans le Calvados et la Basse-Normandie : nous eûmes alors avec lui des rapports et nous pûmes apprécier toute l'étendue de ses connaissances et de son dévouement ${ }^{29}$."

\section{La constitution de la collection d'antiquités}

Entre 1820 et 1850 environ, Northampton constitua une collection composée de vases, de terres cuites, de verres et de miroirs. Les vases, publiés par John Boardman et Martin Robertson en 1979, constituèrent longtemps l'une des plus prestigieuses collections privées d'Angleterre, dispersée seulement en $1980^{30}$. Leur qualité attira l'attention de la communauté savante : plusieurs d'entre eux furent illustrés dans les Monumenti Inediti ${ }^{31}$ avant d'être l'objet d'une conférence donnée par l'archéologue Emil Braun en $1841^{32}$, et dix-huit pièces furent brièvement décrites en 1846 dans l'Archäologische Zeitung ${ }^{33}$.

Le séjour italien semble avoir marqué le début de la collection. À Rome, le marché des antiquités, déjà très florissant, l'était devenu davantage avec la découverte dans les années 1820 des grandes nécropoles d'Étrurie méridionale, Vulci, Cerveteri, Tarquinia, et de nombreux autres sites moins étendus, mais explorés avec autant de frénésie. Selon l'Édit Pacca (1822), le matériel archéologique devait être décrit par les responsables des fouilles et déclaré lors de son arrivée à Rome. Si les musées du Vatican ne souhaitaient pas l'acheter, il pouvait être vendu. La Ville devint donc le cœur du commerce des ors, bronzes et vases découverts dans les tombes étrusques. Le Bullettino de l'Instituto di Corrispondenza Archeologica qui tenait une chronique très précise de la circulation des antiquités nous a transmis le nom des principaux marchands tels Francesco Capranesi (vers 1797- ?), Francesco Depoletti (1779-1854), ou Giuseppe Baseggio $^{34}$ ( ?-après 1871). Ce dernier était en relation étroite avec Lucien Bonaparte et Alexandrine de Bleschamps, princes de Canino, dont il vendit une partie du matériel archéologique découvert sur leurs terres de Vulci. Lord Northampton fit affaire avec Baseggio à plusieurs reprises : la coupe éponyme du Peintre d'Ashby, sans doute restaurée par Depoletti ${ }^{35}$, une amphore attique à figures noires attribuée au Peintre de Madrid ${ }^{36}$, et une amphore panathénaïque découverte lors des fouilles Canino à Vulci ${ }^{37}$ proviennent de sa boutique. Dans les années 1840, Lord Northampton négocia avec Baseggio l'achat d'une vingtaine

26. Op. cit. note 12, p. 20 et $\mathrm{n}^{\circ} 14$.

27. Bulletin monumental, v. 6, 1840, p. 440.

28. A. Schnapp, op. cit. note 2, pp. 339-340.

29. Bulletin monumental, 1851, p. 239.

30. Publiée dans le Corpus Vasorum Antiquorum en 1979 la collection fut vendue chez Christie's en 1980. Voir Greek, Etruscan and South Italian Vases from Castle Ashby, The property of the Marquess of Northampton, Christie's, Londres, Manson \& Woods Ltd., mercredi 2 juillet 1980.

31. Monumenti Inediti dell'Instituto di Corrispondenza Archeologica, I, pl. 21, 9.

32. Bullettino dell'Instituto di Corrispondenza Archeologica, 1841, p. 177.

33. Archäologische Zeitung, 1846, pp. 340-342.

34. Il existe dans les publications plusieurs orthographes de Baseggio : nous avons adopté ici celle qu'il utilisait dans sa signature.

35. CVA Castle Ashby, no 58, p. 22, pl. 36., J. D. Beazley, Attic Red-Figure Vase-Painters, seconde édition 1963 (ensuite abrégé en $A R V^{2}$ ) 455.8. Aujourd'hui au Metropolitan Museum à New-York (1993.11.5). Le passage dans I'atelier de Depoletti est attesté par un dessin du Gerhardscher Apparat XXI, 47.1, Berlin, Altes Museum.

36. CVA Castle Ashby, n० 5, p. 5, pl. 9-10, ABV 329.5.

37. CVA Castle Ashby, n 13, p. 9, pl. 17, ABV 408.1. 
de vases grecs pour le British Museum. C'est probablement en Italie qu'il acheta une amphore à col de la collection Pizzati ${ }^{38}$.

Son retour en Angleterre suivit de peu le début de l'exploration des nécropoles d'Étrurie méridionale, mais ne signifia pas pour autant la fin de l'enrichissement de sa collection, car Lord Northampton poursuivit ses acquisitions lors de ventes publiques à Londres. Il acheta deux vases passés dans les ventes organisées par Giuseppe Baseggio en $1838^{39}$ : une coupe à figures rouges ${ }^{40}$ et une amphore à figures noires ${ }^{41}$ attribuées au Peintre de Nicosthénès. Toutes deux sont inscrites : la coupe porte la signature de Pamphaios et l'amphore celle de Nicosthénès. Quelques années plus tard, en 1849, la collection de vases grecs rassemblés par Thomas Hope (1769-1831) fut vendue aux enchères, et Lord Northampton acheta une amphore signée Andokidès ${ }^{42}$ et un plat signé Epictétos ${ }^{43}$, tous deux provenant des fouilles Canino.

\section{Le choix des vases}

Telle que la reconstituèrent John Boardman et Martin Robertson dans le Corpus Vasorum Antiquorum, la collection de Northampton comptait cent vingt-cinq pièces, un nombre plutôt modeste. Trois vases et neuf fragments sont perdus et mal connus. Nous remarquons d'emblée l'absence d'œuvres de qualité médiocre et le coût probablement élevé de certains vases rares, signés, ou d'amphores panathénaïques, alors très recherchées. La céramique attique était largement dominante (quatre-vingt-sept numéros) mais laissait tout de même la place à un échantillonnage d'autres ateliers : vingt-et-une pièces italiotes, cinq étrusques, trois corinthiennes, une chalcidienne, une daunienne et la célèbre amphore ionienne dite "amphore Northampton ${ }^{44}$ ". Cette distinction moderne par lieux de production recoupe pour partie celle en cours dans les années 1830, où, avec des dénominations différentes et parfois erronées, on distinguait clairement les techniques et les styles des vases ${ }^{45}$. Avec cent numéros, la céramique figurée l'emportait de loin et la variété de ses techniques était illustrée de manière presque complète par des vases à figures noires, figures rouges, figures noires sur fond blanc, des lécythes à fond blanc et un vase surpeint ${ }^{46}$. La collection comptait aussi quatorze vases à vernis noir et deux vases du style de Gnathia. Le panorama ainsi créé laisse supposer une volonté d'exhaustivité. En revanche, du point de vue des formes, le cratère à volutes, le canthare et le rhyton brillaient par leur absence, tandis que coupes et bols, avec un total de vingt-cinq pièces, étaient largement représentés, juste devant les vingt-deux amphores de différents types. Les œnochoés, les lécythes et lécythes aryballisques approchaient chacun la dizaine

38. CVA Castle Ashby, n 66, p. 26, pl. 42. Sur la collection Pizzati, voir Anastasia Bukina, Irina Grogorieva, Ksenia Shugunova et Serguei Khavrine, « Les vases de la collection Antonio Pizzati au musée de l'Ermitage : études scientifiques et perspectives », dans les actes du colloque L'Europe du vase antique : collectionneurs, savants, restaurateurs aux XVIII et XIXe siècles : Paris, INHA, 31 mai - $1^{\text {er }}$ juin 2011, publiés sous la direction de Brigitte Bourgeois et Martine Denoyelle, Rennes, Presses universitaires de Rennes, «Art et société », 2013, pp. 221235.

39. Londres, 13 juin et 13 juillet 1838, Sotheby's. Des exemplaires annotés des catalogues se trouvent à la British Library. Plusieurs des vases présents dans ces deux ventes avaient appartenu auparavant à Francesco Depoletti (1779-1854), marchand et restaurateur de vases grecs très actif à Rome dans les années 1830 et 1840. Le passage de ces vases de Depoletti à Baseggio laisse supposer des ventes entre marchands dans le milieu romain, et dans ce cas peut-être une association pour organiser ces ventes à l'étranger afin de partager les frais et les bénéfices. Ces ventes montrent également la nécessité pour les marchands d'élargir le cercle de leur clientèle, et de proposer des antiquités ailleurs que sur un marché romain saturé.

40. Vente du 13 juin 1838, n 56, CVA Castle Ashby, n 57, pp. 21-22, pl. 38 et 62, ARV 24.7. Beazley Archive 201035. Aujourd'hui à New York, collection Callimanopoulos.

41. Vente du 13 juillet 1838, $n^{\circ} 21$, CVA Castle Ashby, n 18, pp. 11-12, pl. 19.4-8, ABV 221.40., Beazley Archive 302793.

42. CVA Castle Ashby, n० 3, p. 3, pl. 6 et 7.1, ABV 253.2, 293.7, ARV2 1, 2, et 6. Beazley Archive 302217. Aujourd'hui au British Museum $(1980,1029.1)$.

43. CVA Castle Ashby, n 65, p. 25, pl. 41.3, ARV2 77.92., Beazley Archive 200619. Aujourd'hui au Metropolitan Museum (1981.11.10)

44. CVA Castle Ashby, no 1, pp. 1-2, pl. A, 1 à 3, Beazley Archive 1000837. Aujourd'hui dans la collection Niarchos.

45. À ce sujet voir par exemple, E. Gerhard, « Rapporto intorno i vasi volcenti », Annali dell'Instituto di Corrispondenza Archeologica, 1831, pp. 5-218.

46. Cinquante-cinq vases à figures noires, un vase à figures noires sur fond blanc, trois vases bilingues, quarante-deux vases à figures rouges, un vase étrusque surpeint. 
de pièces ${ }^{47}$. Les autres formes, qu'elles soient courantes ou rares, dépassaient rarement les trois exemplaires ${ }^{48}$. L'absence du cratère à volutes, si emblématique du vase grec ${ }^{49}$, est d'autant plus étonnante que le reste de la collection révèle une sensibilité à la forme des vases : la grande variété des profils d'amphores, la présence de plusieurs pièces étonnantes comme le gobelet chalcidien à une anse $^{50}$, le petit vase en forme de chouette ${ }^{51}$ ou la coupe daunienne ${ }^{52}$ démontrent une volonté de ne pas se limiter aux formes canoniques. Enfin, concernant la céramique figurée attique, la période archaïque est largement plus représentée que la période classique : sans doute faut-il y voir une préférence du marquis pour le style retenu du $\mathrm{VI}^{\mathrm{e}}$ siècle et du début du $\mathrm{V}^{\mathrm{e}}$ siècle avant J.-C. Un premier examen dessine donc une collection restreinte et construite autour d'une variété des ateliers et des techniques, et d'un choix de formes attentif, mais non exhaustif.

Nous remarquons également dix-huit pièces inscrites, dont douze signées : les noms d'Andokidès, Epictétos et Pamphaios apparaissent une fois, ceux de Nicosthénès et d'Hermogénès deux fois, et celui de Tléson trois fois ; il faut

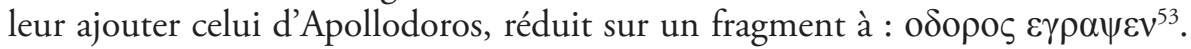
Il ne s'agissait donc pas seulement de collectionner des signatures variées, mais aussi de rassembler des œuvres d'une même main. Cette recherche de signatures est précoce, car les autres exemples connus, telles les collections rassemblées par Auguste van Branteghem et Alfred Bourguignon, datent de la fin du XIX ${ }^{\mathrm{e}}$ siècle $^{54}$. Comme l'a démontré Athéna Tsingarida, ces deux collectionneurs avaient une approche stylistique, et recherchaient des ouvres de peintres en particulier. Dans le cas de Lord Northampton, ces ensembles d'une même main prouvent qu'il s'intéressait à l'identité des potiers et des peintres, et qu'il avait probablement entrevu les possibilités des études que ce champ offrait.

Enfin, un dernier élément semble tout à fait significatif : sur cent vases figurés, trente-deux, soit près du tiers, présentaient des épisèmes ou au moins des boucliers. À titre de comparaison moins de $4 \%$ des vases répertoriés dans la Beazley Archive portent des épisèmes. Image dans l'image, les épisèmes constituent ici une collection dans la collection. Ils furent recherchés pour leur diversité : avanttrains de lion, de panthère, de taureau, arrière-train de cheval, têtes de bœuf et de cheval vues de face, tête de satyre, satyre en course, pégases, cygne, dauphins, biche, chien, pigeons, corbeau, serpents, jambe humaine, gorgonéion, trépieds, roues, disques...; le marquis rassembla un très large éventail des motifs existants. Cette préférence accepte deux explications non exclusives l'une de l'autre : les épisèmes peuvent être compris comme l'héraldique de l'Antiquité, et en tant qu'aristocrate, Lord Northampton y trouvait probablement un intérêt particulier. Cette démarche serait alors à rapprocher de celle du duc de Luynes qui donna dans ses diverses collections une place singulière aux armes et à leurs représentations ${ }^{55}$. Ce choix iconographique peut aussi relever d'un intérêt scientifique du marquis,

47. Douze œnochoés, huit lécythes, sept lécythes aryballisques, sept kyathoi.

48. Quatre hydries, deux cratères à colonnettes, deux cratères en cloche, une pélikè, deux stamnoi, deux aryballes, trois guttus, trois askoi, une épichysis, un unguentarium, deux plats, deux vases en forme de tête humaine, un vase en forme de chouette, une coupe daunienne.

49. Martine Denoyelle, « Le cratère à volutes : fortune antique et fortune moderne » dans les actes du colloques El vaso griego en el arte europeo de los siglos XVIII y XIX, Madrid, Casa Vélasquez, du 14 au 15 février 2005, Madrid, 2008, pp. 89-104.

50. CVA Castle Ashby, no 48, p. 19, pl. 30.1-3, Beazley Archive 1000795. Le vase est qualifié de deep cup dans le CVA, et de stamnos sur le site de la Beazley Archive ; sa faible hauteur $(9,7 \mathrm{~cm})$ et son anse unique justifient la désignation de gobelet.

51. CVA Castle Ashby, n 111, p. 40, pl. 60.5-7, Beazley Archive 1000834, localisation inconnue. 52. CVA Castle Ashby, n' 112, p. 40, pl. 60.8-9, Beazley Archive 1000835, localisation inconnue. 53. Le nom d'Apollodoros fut reconstitué par Hartwig grâce à la comparaison entre ce fragment et une coupe fragmentaire du musée du Louvre (G 139 et G 140), voir Paul Hartwig, Die griechischen Meisterschalen der Blüthezeit des strengen rothfigurigen Stiles, Stuttgart, Berlin, W. Spemann, 1893, pp. 628-630.

54. Athéna Tsingarida, «The Search for the Artist. The van Branteghem and Bourguignon Collections and the Connoisseurship of Greek vases », Sammeln und erforschen. Griechische Vasen in neuzeitlichen Sammlungen, Munich, C.H. Beck, (« Beihefte zum Corpus Vasorum Antiquorum », $\mathrm{n}^{\circ} \mathrm{VI}$ ), 2014, pp. 115-121. Sur la collection van Branteghem, voir également A. Tsingarida, " "Nul ne sait qui n'essaye". Alphonse van Branteghem et sa collection de vases grecs », A. Tsingarida, Donna Kurz, dir. Appropriating Antiquity : collections et collectionneurs d'antiques en Belgique et en Grande-Bretagne au XIXe siècle, Bruxelles, Livre Timperman, 2002, pp. 245-273.

55. Cécile Colonna, De Rouge et de Noir. Les vases grecs de la collection de Luynes, Paris, Gourcuff Gradenigo, 2013, p. 12. 
qui considérait les épisèmes comme une clé d'interprétation de l'iconographie. Cette démarche, qui attribue le pouvoir de faire saisir l'ensemble d'une scène à un détail, n'était pas une exception : le débat des années 1830 autour de l'interprétation de l'épisème d'une amphore du Peintre de Cléophradès en est un autre exemple ${ }^{56}$. En 1847, le marquis publia un article consacré à l'un de ses vases : Observation by the Marquess of Northampton, Pres. R.S., F.S.A, upon a Greek Vase discovered in Etruria, now in his Lordship's possession: bearing the name of the fabricator Nicosthenes ${ }^{57}$. Le texte révèle une très bonne connaissance de l'actualité archéologique, des publications et des collections privées et publiques. Il est consacré à l'étude d'une amphore nicosthénienne provenant de Cerveteri (fig. 2) : dessinée dans l'atelier de Francesco Depoletti où elle fut probablement restaurée ${ }^{58}$, l'amphore fut vendue lors de la vente aux enchères organisée par Giuseppe Baseggio à Londres le 13 juillet 1838 et achetée par le marquis ${ }^{59}$. Le corps porte un décor composé de duels entre hoplites, de boutons de lotus et d'une frise animalière, tandis qu'on voit sur le col une ronde de dauphins, et sur chaque anse une figure masculine imberbe, drapée et tenant une lance. Dans son analyse, le marquis s'intéresse aux noms des potiers et des peintres qu'il appelle manufacturers et suppose à juste titre l'inventivité de Nicosthénès; puis, afin de démontrer le rôle des épisèmes dans l'identification des porteurs de boucliers, il porte l'essentiel de son attention sur les scènes de duel (fig. 3). Saluant la démarche comparable du duc de Luynes, Lord Northampton reconnaît dans l'un des duels le combat d'Achille et d'Hector autour de la dépouille d'Antiloque, et dans l'autre celui d'Achille contre Memnon. Les épisèmes sont un argument essentiel de son raisonnement : le serpent, souvent associé à Achille et à sa protectrice, Athéna, désigne ici ce héros grec; le trépied, un des emblèmes d'Apollon, protecteur des Troyens, permet d'identifier le plus valeureux d'entre eux, Hector ${ }^{60}$.
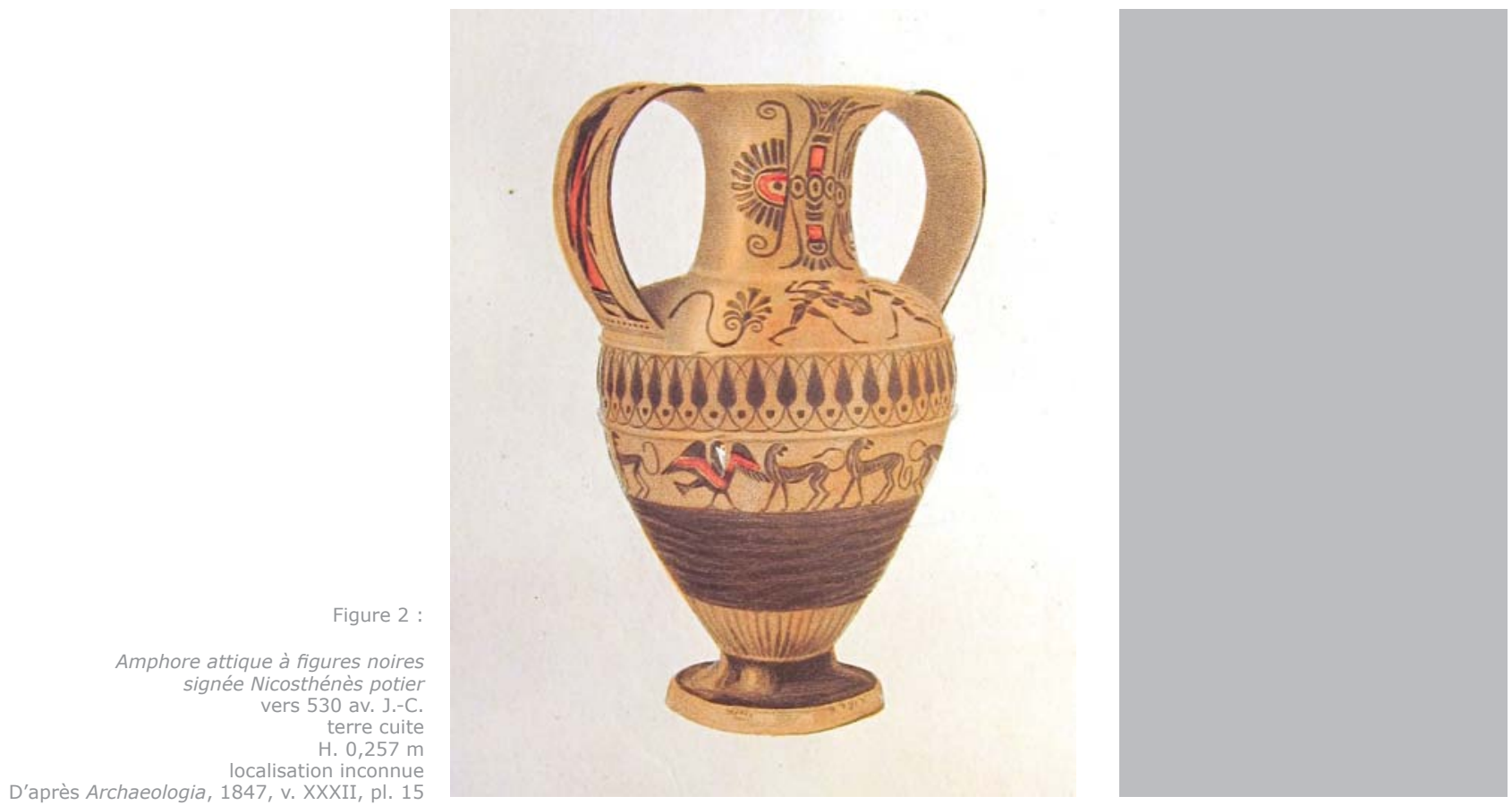

56. Amphore attique à figures rouges attribuées au Peintre de Cléophradès, vers 480 av. J.-C. Würzburg L508, ARV2 182.5 et 1631 , Beazley Archive 201658. Sur le débat concernant l'épisème voir Marie-Amélie Bernard, « Francesco Depoletti (1779-1854), un homme de réseau entre collectionnisme et restauration », B. Bourgeois et M. Denoyelle, op. cit. note 38, pp. 203220.

57. Archaeologia, 1847, vol. XXXII, p. 255-262.

58. Gerhardscher Apparat XI, 17, Berlin, Altes Museum.

59. Elle a été vendue le 2 juillet 1980 chez Sotheby's à Londres, puis repérée sur le marché de l'art à Genève. Sa trace s'est ensuite perdue. ABV 221.44, 225.11, Beazley Archive 302793.

60. Sur les interprétations possibles des épisèmes, nous renvoyons aux travaux de François Lissarrague, notamment « Le temps des boucliers », Giovanni Careri, François Lissarrague, Jean-Claude Schmitt, Carlo Severi, Tradition et temporalité des images, Paris, Éditions de I’EHESS, 2009, pp. 25-35. 
Enfin, cette collection d'épisèmes n'est pas sans rappeler les séries de vases, et particulièrement d'amphores panathénaïques, présentées dans les Monumenti Inediti de l'Instituto di Corrispondenza Archeologica ${ }^{61}$. Là encore, il faut faire le lien entre collection et recherche ; à la suite des très nombreuses découvertes des nécropoles de Vulci, Eduard Gerhard publia une étude dite Rapporto volcente dans laquelle il posa les bases de la céramologie. L'ouvrage comprend, à la suite du texte proprement dit, douze excerpta qui reprennent les idées fortes contenues dans les quelque cent pages précédentes. Gerhard y met en avant l'importance de la comparaison des objets entre eux, soutenue par sa maxime Monumentum artis qui unum vidit, nullum vidit; qui millia vidit, unum vidit ${ }^{62}$. La série d'épisèmes de la collection Northampton répond à ce principe, car elle permet la comparaison immédiate d'une grande variété de motifs et de ceux que l'on retrouve le plus fréquemment.
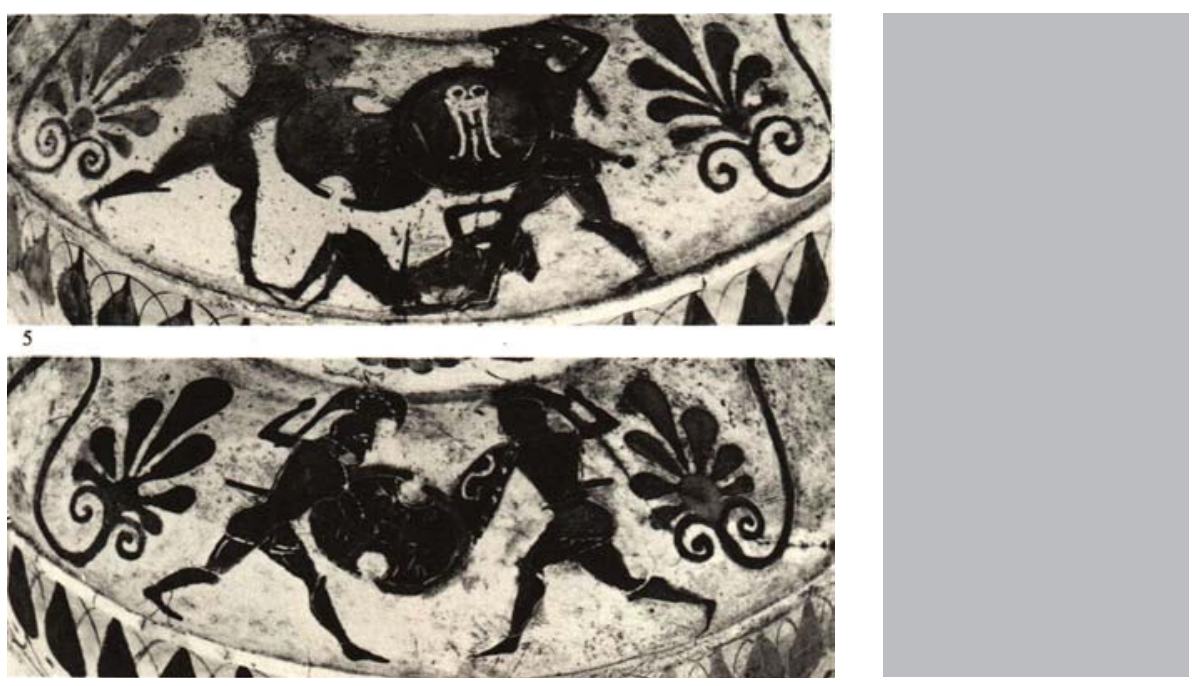

\section{Les fragments}

Lintérêt de Lord Northampton pour les inscriptions et les épisèmes explique la présence dans sa collection d'une quinzaine de fragments dont la majorité sont inscrits ou ornés de boucliers, et choisis, pour cette raison, comme des objets de collection à part entière.

Sur l'un d'eux, on voit la tête et le haut du corps d'un guerrier portant un casque, une lance et un bouclier orné d'une tête de satyre, tandis que de part et d'autre de la figure on lit la signature fragmentaire d'Apollodoros ${ }^{63}$. Un autre fragment montre Dionysos assis entre deux satyres et deux ménades et porte une inscription difficilement lisible ${ }^{64}$; il a longtemps été associé au fragment précédent, et c'est sans doute pour cette raison qu'il est entré dans la collection. Il est particulièrement intéressant dans la mesure où il porte une restauration, incluant, d'après la photographie du Corpus Vasorum Antiquorum, une restitution de l'arrière de la tête de Dionysos ; le satyre de gauche a également subi une intervention, destructive celle-ci : pour des raisons de pudeur, le dessin de son sexe en érection a été grattéte (fig. 4). Ce fragment a donc été traité non pas comme un débris, mais comme un véritable objet de collection : sa restauration est le signe, semble-t-il, de son accès

61. Monumenti Inediti, I, pl. XXII.

62. E. Gerhard, art. cit. note 45, p. 111. «Qui a vu un des monuments de l'art n'en a vu aucun, qui en a vu mille en a vu un. », traduction de l'auteur.

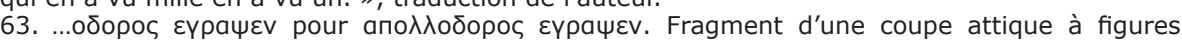
rouges. CVA Castle Ashby, n 64, p. 25, pl. 41.2. ARV 120.4, 1580, Beazley Archive 201006. Aujourd'hui à Bâle, collection H. Cahn (HC 487).

64. Fragment d'une coupe attique à figures rouges. CVA Castle Ashby, 63, p. 25, pl. 41.1. ARV 371.16, 1649, Beazley Archive 203915. Localisation inconnue.

65. Les retouches de pudeur sur les vases grecs sont rares sans être exceptionnelles. Au sujet particulièrement des repeints de pudeur voir B. Bourgeois, " "La fabrique du vase grec. Connaître et restaurer I'antique dans I'Europe des Lumières." Genèse d'un projet », Technè, n 32, 2010, pp. 5-10. 
à la dignité esthétique ${ }^{66}$; elle lui donne un statut autre que celui de curiosité ou de document, car elle suppose que son incomplétude n'exclue pas la délectation.

Figure 4

Détail d'un fragment de coupe à figures rouges attribuée au Peintre de Brygos vers $490-480$ av. J.-C. $0,270 \mathrm{~m}$ localisation inconnue D'après J. Boardman M. Robertson, Corpus Vasorum Antiquorum Great Britain, fascicule $15 \mathrm{pl}$. 41.1.
. Wilkins

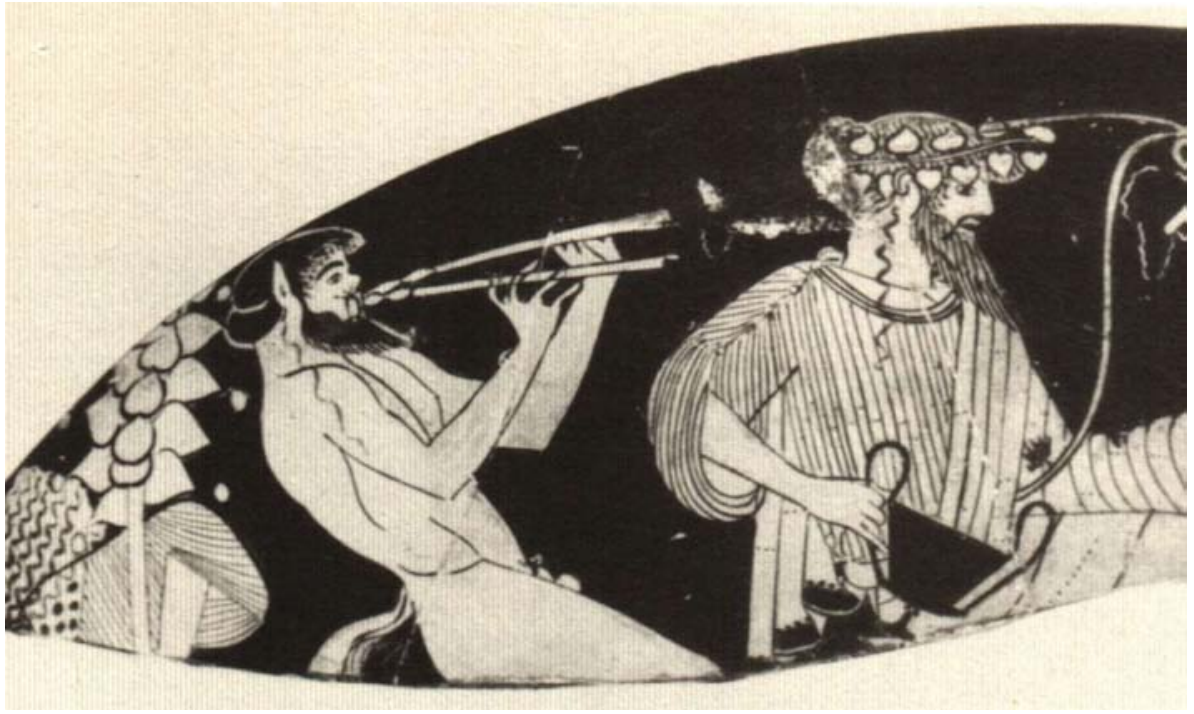

Les autres fragments de la collection semblent avoir été choisis avec soin : on voit, sur l'un, un épisème avec deux dauphins tête-bêche ${ }^{67}$ (fig. 5), sur l'autre, un bouclier portant une biche de profil ${ }^{68}$, sur un troisième deux guerriers dont les boucliers sont ornés d'un pégase, et d'un satyre en course ${ }^{69}$. Il faut leur ajouter plusieurs fragments qui n'étaient plus dans la collection lors de son étude par J. Boardman et M. Robertson. Certains, connus par des descriptions et des dessins, portent aussi des épisèmes : le Deutsches Archäologisches Institut conserve une représentation d'un fragment où un personnage masculin tient un bouclier sur lequel un homme chevauche un dauphin ${ }^{70}$.

-La présence de fragments dans la collection s'explique donc principalement par un choix iconographique plus que stylistique ou technique comme cela semble être le cas pour d'autres collections où les fragments tiennent une bonne place ${ }^{71}$. Elle doit aussi être mise en relation avec l'avancée de l'archéologie dans les années 1830 : dans les excerpta de son Rapporto volcente, Eduard Gerhard plaça cette phrase : Leonem ex ungue, Volcentes ex voce, Graecorum artem ex testa cognosces $^{72}$. Il ne paraît pas indifférent que la maxime Leonem ex ungue apparaisse ici en si bonne place, car elle était associée par les contemporains aux travaux de Cuvier sur l'étude des ossements fossiles d'animaux disparus :

«Épars, brisés, défigurés, les ossements fossiles ne présentent souvent à l'œil peu exercé qu'un fragment informe [...] C'était le cas de réaliser l'ancien proverbe : Ex ungue leonem! (Une griffe suffit pour reconnaitre le lion).

66. Nous empruntons cette expression à Nathalie Heinich, voir N. Heinich, « L'accès à la dignité biographique : les premières mentions de peintres dans les dictionnaires biographiques français. », dans Matthias Waschek dir. Les Vies d'Artistes, Paris, musée du Louvre Ed., École nationale supérieure des beaux-arts, 1996, p. 196 sq.

67. Fragment d'amphore attique à figures noires, CVA Castle Ashby, n 15, pl. 18.6, pp. 10-11. Beazley Archive 27. Localisation inconnue.

68. Fragment d'amphore attique à figures noires, CVA Castle Ashby, n 16, pl.18.7, p. 11. Beazley Archive 30. Localisation inconnue.

69. Fragment d'amphore (?), CVA Castle Ashby, n 90, pl. 55.1, p. 35. Beazley Archive 1000810. Ce dernier est conservé au British Museum (inv. 1989,0307.1) et son authenticité est mise en doute par le musée. S'il s'agit bien d'un faux, (ce que confirmerait la vue simultanée des deux faces ornées des boucliers), c'est intéressant quant à l'adaptation du marché à la demande des collectionneurs.

70. Archäologische Zeitung 1846, 342, n 12. Archäologischer Anzeiger 1977, 246, n 75, fig. 100. Le dessin est visible à cette adresse : http://arachne.uni-koeln.de/item/marbilder/4832544.

71. Les collections van Branteghem et Bourguignon, citées plus haut, comptaient un nombre important de fragments recherchés parce qu'ils portaient une signature ou parce qu'ils présentaient un intérêt du point de vue du style. Comme l'a fait remarquer Athéna Tsingarida, les collections incluant des fragments se multiplient parallèlement aux recherches sur les personnalités des peintres de vases. Voir A. Tsingardia, 2014, op. cit. note 54, p. 119.

72. E. Gerhard, op. cit. note 45, p. 111. La maxime signifie : « Tu reconnaîtras le lion à son ongle, les habitants de Vulci à leur vocable, l'art des Grecs à ses vases. », traduction de l'auteur. 
C'est en effet ce proverbe qui est devenu, par les savantes recherches de

$\mathrm{M}$. Cuvier, une vérité importante, une grande maxime scientifique ${ }^{73}$.»
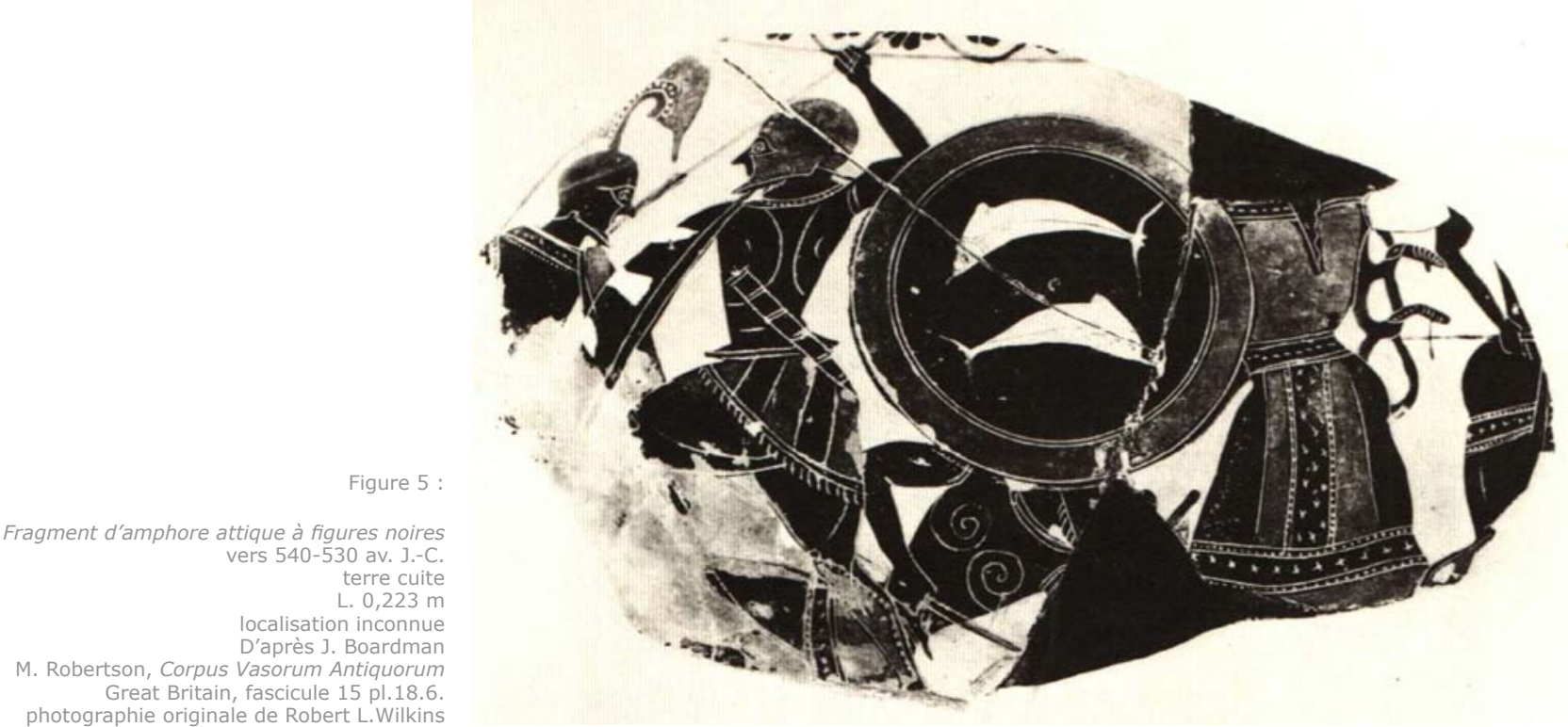

Gerhard, qui sa vie durant s'efforça de faire de l'archéologie une "sœur jumelle » de la philologie ${ }^{74}$, fit-il ici une référence volontaire à Cuvier ? Il semble présomptueux de l'affirmer, mais si cela s'avérait, en découlerait alors un parallèle intéressant entre la paléontologie et l'archéologie, entre les sciences de la nature et les sciences de l'homme ; quoi qu'il en soit, Lord Northampton, membre de l'Instituto di Corrispondenza Archeologica fondé par, entre autres, Eduard Gerhard et de la Royal Society, à laquelle appartint Cuvier, ne pouvait ignorer les travaux de l'un ni de l'autre de ces savants. N'a-t-il pas adopté dans la constitution de sa collection de vases grecs une démarche naturaliste semblable à celle qui lui faisait accorder son attention aux minéraux et aux fossiles ? Cette approche est particulièrement valable pour les vases grecs dont les formes répétitives peuvent être facilement reconnues d'après des fragments. Nous pouvons donc supposer que les deux collections avaient une assise intellectuelle commune.

Sans surévaluer le rôle du marquis de Northampton dans la science du début du XIX ${ }^{e}$ siècle, il faut cependant constater à quel point il fut sensible aux développements auxquels il assista, et qu'il accompagna grâce à ses fonctions importantes dans les sociétés savantes. Il laissa une trace discrète, mais néanmoins bien marquée dans les disciplines auxquels il s'intéressa : la minéralogie a sa comptonite, roche volcanique qu'il découvrit sur les pentes du Vésuve, la paléontologie a son Regnosaurus northamptoni, dinosaure herbivore ainsi nommé par Gideon Mantell en 1848, enfin la céramologie compte dans ses rangs le Peintre de Northampton, le Peintre d'Ashby, du nom de la demeure du marquis, ainsi que l'amphore Northampton. Au-delà de ces dénominations qui sont autant d'hommages, sa collection de vases grecs mérite un nouveau regard : elle ne fut pas seulement « la plus belle collection privée d'Angleterre " mais aussi le véritable laboratoire d'idées d'un homme qui comprit et encouragea les avancées de scientifiques de son temps.

73. Conrad Malte-Brun, Mélanges scientifiques et littéraires de Malte-Brun, ou Choix de ses principaux articles sur la littérature, la géographie et I'histoire, recueillis et mis en ordre par M. J. Nachet, 1828, v. 3, p. 392. Voir également A. M. Madrolle, Tableau de la dégénération de la France, des moyens de sa grandeur et d'une réforme fondamentale dans la littérature, la philosophie, les lois et le gouvernement, 1834, p. 378 : "Donnez-moi une partie quelconque du corps, disait Cuvier, et je vous dirai le corps tout entier : ex ungue leonem. »

74. A. Schnapp, op. cit. note 2, p. 371. 


\section{L'auteur}

En cinquième année de thèse à l'École du Louvre et à l'Université Paris 1 Panthéon Sorbonne, Marie-Amélie Bernard a été d'octobre 2010 à septembre 2014, chargée d'études et de recherche à l'INHA dans l'axe " Histoire de l'art antique, histoire de l'archéologie » dirigé par Martine Denoyelle. Elle est depuis le $1^{\text {er }}$ octobre 2014 et pour six mois boursière du Gratuiertenkolleg Materialität und Producktion à l'université Heinrich Heine, Düsseldorf. Sa thèse dirigée par Brigitte Bourgeois et Alain Schnapp porte sur « Francesco Depoletti (1779-1854), artiste, marchand et restaurateur de vases grecs » et croise des approches d'histoire de l'archéologie, d'histoire de la restauration et d'histoire des collections. 